\title{
Ruling out underlying abnormalities in patients with febrile UTI
}

Urinary tract infections (UTIs) are usually mild; however, in patients with fever, the presence of other urological conditions or complications needs to be assessed. Radiological evaluations are frequently used in patients with febrile UTI to facilitate the diagnosis of any underlying urological anomaly. A new study presents a clinical rule that can predict which patients with febrile UTI will benefit from investigative radiology-potentially reducing the number of unnecessary imaging studies without loss of clinically relevant information. "In our clinical experience, we often did a radiological survey to exclude urological underlying abnormalities, but the yield was very low," notes first author Cees van Nieuwkoop, Leiden University, The Netherlands.

In their prospective observational study, van Nieuwkoop and colleagues evaluated adults with febrile UTI from eight emergency departments (five of which constituted the derivation cohort and

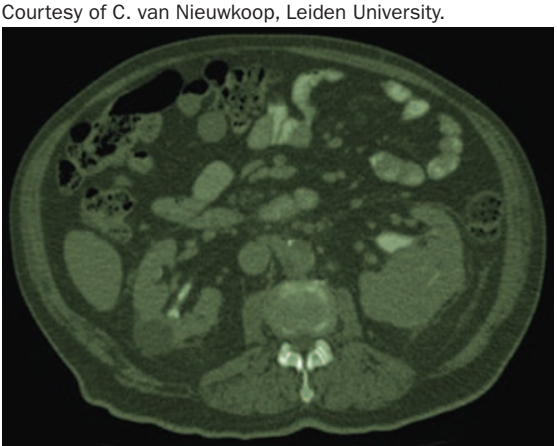

three the validation cohort) from centers in The Netherlands.

Using data from 245 of 346 patients who underwent imaging (in total, 268 ultrasonographies and 81 CT scans), the researchers evaluated clinically relevant radiological findings and classified patient outcomes to derive a clinical rule-history of urolithiasis, urine $\mathrm{pH} \geq 7$ and/or renal insufficiency (estimated GFR, $\leq 40 \mathrm{ml} / \mathrm{min} / 1.73 \mathrm{~m}^{2}$ ) — that was capable of predicting the need for radiological imaging. This rule could exclude any nonurgent or urgent urological disorders (pyonephrosis, renal stones or abscess) with precision and was validated in an independent cohort of 131 patients.

"Applying such a rule into clinical practice might save about $40 \%$ of radiological procedures," adds van Nieuwkoop, and "could replace the previous recommendation to obtain radiological imaging of the urinary tract in cases of persisting fever for 3 days despite active microbial treatment." The authors now plan further research to validate this new prediction rule in different settings and cohorts, as well as evaluate its cost effectiveness and safety in daily clinical practice.

\section{Katrina Ray}

Original article van Nieuwkoop, C. et al. Predicting the need for radiologic imaging in adults with febrile urinary tract infection. Clin. Infect. Dis. 51, 1266-1272 (2010) 